REVISTA PROYECCIONES $\mathrm{N}^{\circ} 10: 35-56$

Diciembre 1985 - ISSN 0716-0917

Jornada de Matemáticas, Agosto 1985.

\title{
ESPACIOS DE APLICACIONES LINEALES
}

Dr. Samuel Navarro*

31. CONTINUIDAD DE APLICACIONES LINEALES.

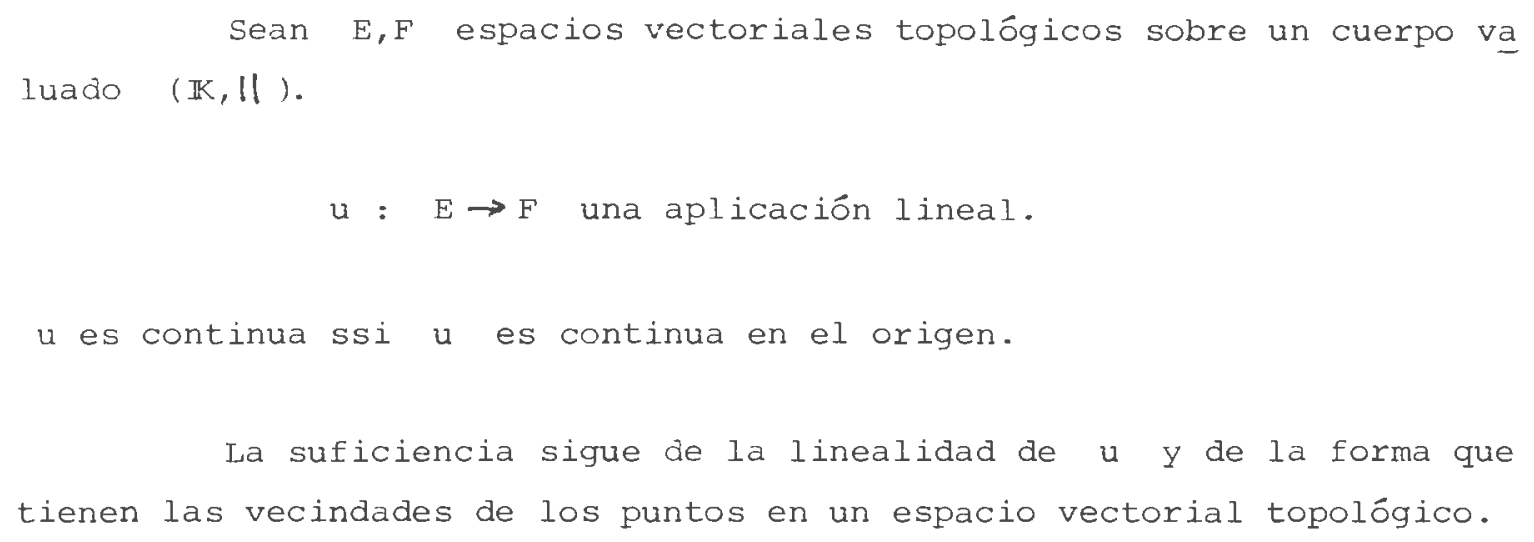

* Departamento de Matemáticas, Universidad de Santiago, Santiago, Chile. 
Observación:

Si $u$ es continua en el origen $u$ es uniformemente continua. Las uniformidades consideradas son las correspondientes a la topología de $E$ y $F$ respectivamente.

$$
\left(\left\{u_{v}=\{(x, y) / x-y \in v\} / v \in B, B \text { una base de } 0\right\}\right.
$$

es una base de la uniformidad).

Proposición:

Si E,F son e.v.t. $y \quad F$ es completo $y$ Hausdorff entonces to da función lineal continua.

$$
\text { u : } E \rightarrow F
$$

tiene una única extensión

$$
\check{L}: \tilde{E} \rightarrow F
$$

donde $\stackrel{\sim}{E}$ es cualquier espacio vectorial topológico donde $E$ es denso.

Demostración:

Ver Tadquir Husain Ch. V, Th 9 para el caso general de una fun ción uniformemente continua.

Corolario:

Con las condiciones de la proposición anterior, si $\hat{E}$ es la completación de $E$ entonces existe una única extensión de $u$ a $\hat{E}$. 
En el caso que los espacios E y F tengan topologías localmen te convexas, el resultado anterior se puede enunciar vía seminormas.

Proposición:

Sean E,f espacios vectoriales topológicos con topologías localmente convexas. Si la topología de $\mathrm{E}$ es generada por la familia de seminormas $s, s=\left\{p_{i} / i \in F\right\}$ entonces $s i u: E \rightarrow F$ es lineal, $u$ es continua si y sólo si $\forall q$; q seminorma sobre $F$, existe $J \subseteq I$, $|J|<\infty \quad y \quad \lambda>0$ tal que $q(u(x)) \leqslant \lambda \sup _{i \in J} p_{i}(x) \forall x \in E$.

\section{Demostración:}

$(\Rightarrow)$ Sea $q$ s-n continua en $F$ entonces para
$U=\{y \in F / q(y) \leqslant I\}$ existe $\left\{V_{i}\right\} i=1, \ldots, n$ donde
$V_{i}=\left\{x \in E / p_{i}(x) \leqslant \alpha_{i}\right\}$ tal que $u\left(\underset{i=1}{n} v_{i}\right) \subseteq U$

si $\alpha=\min _{i}\left\{\alpha_{i}\right\}$ entonces $\sup p_{i}(x) \leqslant \alpha$ implica $u(x) \in V$ i.e. $q(u(x)) \leqslant 1$, de aqui se obtiene que

$$
\begin{gathered}
q(u(x)) \leqslant \alpha^{-1} \cdot \sup _{i} p_{i}(x) \forall x \text { entonces } \lambda=\alpha^{-1}, \\
J=\{1, \ldots, n\} \text { sirven }
\end{gathered}
$$

$\Leftrightarrow$ Sea $V$ una vecindad de $O$ en $F$, equilibrada $y$ convexa, consideramos el funcional de Mimkowski asociado a $V$, este resulta ser una seminorma continua sobre $F$.

$$
\begin{aligned}
& \text { Luego } \exists \lambda>0, \exists\left\{p_{i}\right\} i=1, \ldots, n \subseteq s \text { tq } u(U) \subseteq V \text { donde } \\
& U=\left\{x / c \cdot p_{i}(x) \leq 1, i \in J\right\}
\end{aligned}
$$


Corolario:

$$
\begin{gathered}
\text { Sean } E, F \text { espacios normados } \\
\mathrm{U}: \mathrm{E} \rightarrow \mathrm{F} \text { lineal }
\end{gathered}
$$

$\mathrm{u}$ es continua ssi $3 \quad \lambda>0$ tq ||$u(x)|| \leqslant \lambda|| x|| \forall x \in E$

Definición:

Dados E,F espacios vectoriales topológicos una función lineal $\varphi: E \rightarrow F$ es un homomorfismo topológico si $\varphi$ es continua $y$ es abierta como función de $\mathrm{E}$ en $\varphi(E)$

Observación:

1) Las funciones lineales canónicas del espacio en un cuocien te $y$ de un subespacio en el espacio, son ejemplos de homomorfismos topológicos.

2) Toda función lineal $\varphi$ de un espacio $E$ en $F$, puede des componerse en función de homomorfismos del tipo mencionado en 1); si $\mathrm{K}=\varphi^{-1}(0), v: E \rightarrow E / K$ la función canónica, $i: \varphi(E) \rightarrow F$ la inclusión canónica entonces

$$
\varphi=i \circ \varphi_{0} \circ v
$$

donde $\varphi_{0}: \mathrm{E} / \mathrm{K} \rightarrow \varphi(\mathrm{E})$ es el isomorfismo lineal canónico

$$
\text { 3) con las condiciones de 2); }
$$
a) $\varphi$ es abierta ssi $\varphi_{0}$ es abierta
b) $\varphi$ es continua ssi $\varphi_{0}$ es continua 
Proposición:

Si $E, F$ son e.V.Y., $\varphi: E \rightarrow F$ lineal entonces son equivalentes.

a) $\varphi$ es un homomorfismo topológico

b) si $\mathcal{V}$ es una base de 0 en $E$ entonces $\varphi(\mathcal{V})$ es una base de 0 en $\varphi(E)$

c) $\varphi_{0}$ es un isomorfismo topológico

Proposición:

Sea $E$ e.v.t. tal que $\operatorname{dim}(\varphi(E))<\infty, \varphi(E)$ Hausdorff entonces son equivalentes
a) $\varphi$ es continua
b) $\varphi^{-1}(0)$ es cerrado en $E$
c) $\varphi$ es un homomorfismo topológico

Demostración:

b) $\Rightarrow$ c) Si $\varphi^{-1}(0)$ es cerrado entonces $E / \varphi^{-1}(0)$ es Hausdorff de dimensión finita, $\varphi_{0}: \mathrm{E}_{\varphi^{-1}}(\mathrm{O}) \rightarrow \varphi(\mathrm{E})$ es un isomorfismo entonces $\varphi$ es un homorfismo topológico

Corolario:

Toda forma lineal continua en un espacio vectorial topológico es un homomorfismo topológico 


\section{\$2. TEOREMA DE LA APLICACION ABIERTA - GRAFICO CERRADO.}

La última proposición dice que: toda función lineal continua cuyo rango es de dimensión finita $\left(\mathrm{T}_{2}\right)$ es abierta.

La inquietud es saber si se puede caracterizar los espacios donde este enunciado se verifica

Proposición:

Sean E,F e.v.t. metrizables completos.

$\varphi: E \rightarrow F$ una función lineal continua tal que $\varphi(E)$ es denso en F. Entonces una de las proposiciones siguientes se verifica

i) $\varphi(E)$ es de primera categoría

ii) $\varphi(E)=F \quad y \quad \varphi$ es abierta ( $\varphi$ homomorfismo topológico)

Esta proposición se debe a Banach y su demostración está en [Banach] Capítulo 3 Teor. 3. Es también conocido como el Teorema de Banach - Schauder.

Corolario 1:

Sean E,F e.v.t, metrizables, completos.

$\varphi: E \rightarrow F$ una función lineal y continua, entonces $\varphi$ es un homomorfismo topológico.

ssi $\varphi(E)$ es cerrado en $F$. 
Corolario 2:

Si E es un e.v.t. metrizable y completo con dos topologías comparables entonces las topologías son iguales.

Corolario 3 :

Si E es un e.v.t. metrizable, completo y es la suma directa algebraica de dos subespacios cerrados entonces es la suma directa topo lógica de esos subespacios.

Después del resultado de Banach, en 1949 Diendonne y Schwartz extendieron el corolario 1 a un caso más general.

Proposición:

Sean $E$ un LF-espacio localmente convexo y $F$ un espacio localmente convexo Frechet (o LF) entonces toda aplicación lineal continua $\varphi: E \rightarrow F$ sobre, es un homomorfismo topológico.

La siguiente proposición está directamente ligada a la de la aplicación abierta y es conocida como el teorema del gráfico cerrado.

Teorema:

Sea E,: e.V.t. metrizables, completos $\varphi: E \rightarrow F$ lineal
entonces $\varphi$ es continua ssi el gráfico de $\varphi$ es cerrado en L $x$ M. Demostración:

$\Leftrightarrow$ Si graf. $\varphi$ cerrado es un subespacio completo metrizable de $E x F$. La función $f: \operatorname{graf} \varphi \rightarrow E$, tal que $f((x, \varphi(x)))=x$ es biyectiva, lineal y continua luego abierta entonces la función 
$g: E \rightarrow$ graf $\varphi, g(x)=(x, \varphi(x))$ es continua luego $\varphi$ es continua.

Sobre el teorema de la aplicación abierta y del gráfico cerrado existen abundantes intentos por obtener caracterizaciones óptimas de los espacios en que se verifica, ver por ejemplo: T. Husain, S. Iyahen, V. Ptak, S. Ohwaki y Mahowald. Conociendo teoría de dualidad se puede estudiar los espacios B-completos o espacios de ptak donde hay un considerable avance en la caracterización buscada.

\section{§3. FORMAS LINEALES CONTINUAS.}

¿Cuántas formas lineales continuas existen sobre un espacio vectorial topológico?

Por lo menos el conjunto no es vacío ya que si $E$ es un e.v.t. $y$ denotamos por $E^{\prime}=\left\{f: E \rightarrow \mathbb{E} / f\right.$ lineal y continua ${ }^{\prime}$ se tiene que $0 \in E^{\prime}$ (0 la función constante igual a 0). E' se llama dual topológico de $E$.

Existen espacios cuyo dual topológico no es más que $\{0\}$, (ejemplo a continuación) entonces ¿cuándo $E^{\prime}$ no es trivial?.

Ejemplo:

$$
\begin{aligned}
& \text { El espacio } L^{\mathrm{P}}, 0<\mathrm{P}<1 \text { tiene dual trivial, i.e. } \\
& \left(\mathrm{L}^{\mathrm{P}}\right)^{\prime}=\{0\} \text { si } 0<\mathrm{p}<1 .
\end{aligned}
$$

Sea I intervalo unitario, $\mu$ la medida de Lebesque en I sea -P el espacio vectorial real de todas las funciones reales, $\mu$ medibles tales que $|f|^{p}$ es $\mu$-integrable. 
Denotamos por $L^{p}$ el cuocieciente de $\mathcal{L}^{p}$ por las funciones nulas casi en todas partes (c.t.p.).

Definimos $s: L^{p} \rightarrow \mathbb{R}$ por $s(f)=\left(\int_{I}|f|^{p} d \mu\right)^{1 / p} s$ es una seudonorma en $L^{P}\left(L^{p}, s\right)$ es un e.v.t. Hausdorff, completo.

Caracterizando la topología por vecindades obtenemos $\left\{\mathrm{U}_{\varepsilon}=\left\{\mathrm{f} /\left(\mathcal{S}_{\mathrm{I}}|\mathrm{f}|^{\mathrm{P}} \mathrm{d} \mu\right)^{1 / \mathrm{P}}<\varepsilon\right\}\right\}_{\varepsilon>0}$ es una base de vecindades de 0 . La siguiente desigualdad se tiene:

$$
|| f+g \|_{p} \leqslant 2 \frac{1-p}{p}\left(\|f\|_{p}+\|g\|_{p}\right)
$$

(desigualdad de Minkowski para $0<\mathrm{p}<1$ )

Con esto se prueba que

$$
2^{-1 / p} u+2^{-1 / p} u \subseteq U
$$

si $U$ es una vecindad de 0 de la base indicada. Así $L^{P}$ es efectiva mente un e.v.t.

Vamos a demostrar que si $\varphi \in\left(L^{p}\right)$ ' entonces $\varphi=0$

Suponemos que $\varphi \in\left(L^{p}\right), y \varphi_{\ell} \not 0$ entonces existe $f_{0} \in L^{p}$ tq $\varphi\left(f_{0}\right)=1$

Sea $\alpha \in[0,1]$, definimos

$$
\begin{aligned}
& g_{\alpha}^{(1)}(t)=\left\{\begin{array}{ll}
f_{0}^{(t)} & t \in[0, \alpha] \\
0 & t \in] \alpha, 1]
\end{array} \quad y\right. \\
& g_{\alpha}^{2}(t)=f_{0}(t)-g_{\alpha}^{1}(t)
\end{aligned}
$$


La función de $\alpha,|| g_{\alpha}^{(1)}||_{p}^{p}=\int_{0}^{\alpha}\left|f_{0}\right|^{p} d \mu$ es continua $y$ creciente. (Crece hasta $\left.\left\|f_{0}\right\|_{p}^{p}\right)$ luego existe $\alpha_{0}$ tal que

$$
\left\|g_{\alpha}^{(1)}\right\|_{p}^{p}=\left\|g_{\alpha}^{(2)}\right\|_{p}^{p}=\frac{1}{2}\left\|f_{0}\right\|_{p}^{p}
$$

como $\left|\varphi\left(f_{0}\right)\right|=1$ entonces

$$
\left|\varphi\left(g_{\alpha}^{(1)}\right)\right| \geqslant \frac{1}{2} \quad 0 \quad\left|\varphi\left(g_{\alpha}^{(2)}\right)\right| \geqslant 1
$$

verifique

$$
\text { Anotamos } f_{1}(t)=2 g_{\alpha_{0}}^{(i)}(t) \text { para } i=1 \quad \delta \quad i=2 \text { según }
$$

$$
\text { Claramente }\left|\varphi\left(f_{1}\right)\right| \geqslant 1 \text { pero }|| f_{1}||_{p}=2^{1}-\frac{1}{p}|| f_{0}||_{p}
$$

intunces obtenemos una sucesión $\left\{f_{i}\right\}$ que verifica

$$
\begin{gathered}
\qquad\left.\varphi\left(f_{i}\right)|\geqslant 1 \quad y \quad|\left|f_{i}\right|\right|_{p}=2^{i\left(1-\frac{1}{p}\right)} \|\left. f_{0}\right|_{p} \\
-\left.\right|_{i} \|_{p} \rightarrow 0 \text { y }\left|\varphi\left(f_{i}\right)\right| \geqslant 1 \quad \forall i
\end{gathered}
$$

. $\varphi$ es continua, esto es una contradicción luego no existe una $\geq 2$.

El ejemplo muestra un espacio vectorial topológico metrizable con dual trivial. El siguiente lema presenta un medular resultado que tiende a la caracterización de los espacios con "buen" dual.

Lema:

Sea E un e.v.t. real, Hausdorff y de dimensión mayor o igual que 2. Entonces si $C \subseteq E$ es un abierto convexo que no contiene el ori gen, existe un subespacio $S$ unidimensional de $E$ que no intersecta $\mathrm{C}$. 
Demostración:

Sea $B$ un subespacio bidimensional de $E$ si $B \cap C=\Phi$

listo.

Si $B \cap C=D \neq \phi$ entonces $D$ es abierto, convexo y $O \notin D$.

Como $\mathrm{B}$ es isomorfo a $\mathbb{R}^{2}$, consideramos $\mathrm{D} \subseteq \mathbb{R}^{2}$.

Sea $\varphi: D \rightarrow \mathbb{R}^{2}, \varphi(x, y)=\left(\frac{x}{r}, \frac{y}{r}\right)$ donde $r=\left(x^{2}+y^{2}\right)^{1 / 2}$

entonces $\varphi(D)$ está en el círculo unitario de $\mathbb{R}^{2}$

$\varphi(D)$ es conexo y abierto

$\varphi(D)$ es un arco abierto con ángulo central menor o igual a $\Pi$.

luego existe una recta que pasa por el origen que no intersecta $\varphi$ (D).

Teorema:

Sea E un espacio vectorial, M una variedad lineal de E, C un conjunto convexo, abierto no vacio que no intersecta $M$. Entances existe un hiperplano cerrado que contiene a 11 y no intersecta a $\mathrm{C}$.

Demostración:

Suponemos que $M$ es subespacio, consideraremos

$\sigma=\{\mathrm{S} \subseteq \mathrm{L} / \mathrm{S}$ subespacio real cerrado, $\mathrm{M} \subseteq \mathrm{S}, \mathrm{S} \Omega \mathrm{C}=\phi\}$

por zorn, o tiene elemento maximal $\mathrm{H}_{\mathrm{O}}$.

Usamos el lema anterior para demostrar que el cuociente $E_{0}{ } \mathrm{H}_{\mathrm{O}}$ (E espacio real asociado a E) tiene dimensión uno. Luego $\mathrm{H}_{0}$ es el 
hiperplano buscado, caso $\mathbb{R}$ ). Este resultado se extiende naturalmente al caso $\mathbb{c}$.

Corolario:

Si $E$ es un e.v.t. entonces existe una forma lineal continua $f \neq 0$ ssi $E$ contiene un conjunto $C$, convexo no vacío, abierto y distinto de $\mathrm{E}$.

Jemostración:

$\Leftrightarrow$ Si $f \neq 0$ es lineal continua.

$$
C=\{x /|f(x)|<1\}
$$

es abierto, convexo, no vacío y $\neq \mathrm{E}$

$(\because=$ Sup. C convexo, $\neq \phi, \neq \mathrm{E}$, abierto. Entonces si $x_{0} \notin \mathrm{C}$ existe un hiperplano cerrado $\mathrm{H}$ tal que $\mathrm{H} \cap \mathrm{C}=\phi \mathrm{y}_{\mathrm{O}} \in \mathrm{H}$, pero $\mathrm{H}$ es hiperplano cerrado ssi existe $f$ lineal y continua $y$ $\alpha \in \mathbb{K}$ tal que $H=\{x / f(x)=\alpha\}$. Si $\alpha \neq 0$ listo, si

$$
\alpha=0 \text { entonces } f(c) \neq 0 \quad \forall c \in C, \text { si no } C \cap H \neq \phi
$$

Este teorema o su corolario, son conocidos como el Teorema de Irahn - Banach (forma geométrica). Este teorema es conocido también como Teorema de Mazur, fue demostrado por Mazur posterior al teorema de HahnBanach que es enunciado en la forma siguiente (llamada analítica). 
Teorema:

Sea $E$ un e.v., p una seminorma en $E$ y $M$ un subespacio de E. Si $f$ es una forma lineal sobre $M$ tal que $|f(x)| \leqslant p(x)$, para cada $x \in M$, entonces existe una forma lineal $f_{1}$ que extiende $f a$ todo $E$ y tal que $\left|f_{1}(x)\right| \leqslant p(x)$, para todo $x \in E$.

Este teorema no es exactamente el enunciado por Hahn ni Banach, el enunciado clásico es en espacios normados.

$\operatorname{Si}(E,||||)$ es normado, $M \leqslant E$, $f$ forma lineal en $M$
tal que $|f(x)| \leqslant|| x||, x \in M$ entonces existe $f_{I}$ extensión de $f$ a $E$ tal que $\left|f_{1}(x)\right| \leqslant|| x||, \quad(x \in E)$.

Existe una gran variedad de enunciados del teorema de HahnBanach, dependiendo del área en que se trabaja o del tipo de problemas que se analiza. En F. Tréves, Ch. 18, se puede encontrar enunciados para aplicaciones en problemas de aproximación, problemas de existencia - problemas de separación.

Una historia del teorema de Hahn-Banach puede encontrarse en Bourbaki (Note Historique) o Monna.

Los espacios localmente convexos, son e.v.t. donde el teorema de $\mathrm{H}-\mathrm{B}$ se verifica, luego tiene un espacio dual digno de considerar (Teoría de dualidad tiene aquí sentido). 


\section{§4. TOPOLOGIAS EN EL ESPACIO DE FUNCIONES LINEALES.}

Dado $E, F$ e.v.t. localmente convexos denotamos por $\mathcal{L}(E, F)$ el espacio de todas las aplicaciones lineales y continuas de $E$ en $F$.

Suponemos que $G$ es una familia de conjuntos acotados de $E$, tal que la unión de todos los conjuntos de $G$ es total en E. (i.e. la cápsula lineal de la unión es densa en $E$ ). Suponemos además que $\mathcal{V}$ es una base de vecindades de 0 en $F$.

Definimos los conjuntos

$$
M(S, V)=\{f \in \hat{\alpha}(E, F) / f(S) \subseteq V\}
$$

donde $s \in G$ y $\quad v \in \mathcal{V}$.

La familia $\{M(S, V)\}_{G, V}$ es una base de vecindades de 0 , para una única topología localmente convexa Hausdorff en $\mathcal{L}(E, F)$. Esta topología ae llama topología de la convergencia uniforme sobre los conjuntos $\because$ de $G$ o brevemente la $G$ - topología.

El par $(E, E, F), G$ - topología) se anota $\mathcal{L}_{G}(E, F)$ o también $\alpha_{x}(E, F)$ donde $x$ es un símbolo que indica la característica de los conjuntos de $G$

Ejemplos de $G$ - topologías en $\mathcal{L}(E, F)$.

1) Si $G$ es la familia de todos los subconjuntos finitos de E. La topología se llama: Topología de la convergencia puntual o también topología simple.

2) Si $G$ es la familia de todos los subconjuntos compactos, convexos, equilibrados de $E$. 
La topología so llama topología de la convergencia sobre los compactos, convexos, equilibrados.

3) Si $G$ es la familia de todos los subconjuntos precompactos de E. La G - topología es la topología de la convergencia precompacta.

4) Si $G$ es la familia de todos los subconjuntos acotados de E. La G - topología es la topología de la convergencia acotada.

Observación:

La $G$ - topología es idéntica con la $\bar{G}$ - topología si denota una familia saturada de $\mathbf{G}$.

Una familia $S \neq\{\phi\}$ de conjuntos acotados de $E$ es saturada si contiene todos los subconjuntos de sus miembros, todos los múltiplos por escalares de sus miembros y todas las cápsulas equilibrada, convexa, cerrada de las uniones finitas de sus miembros.

Se puede también caracterizar la $G$ - topología vía seminormas.

Si $P=\left\{P_{\alpha} / \alpha \in A\right\}$ es una familia de seminormas que genera la topología de $F$, entonces la familia

$$
=\left\{p_{S, \alpha} / S \in G, \alpha \in A\right\}
$$

donde $p_{S, \alpha}(\varphi)=\sup _{S} p_{\alpha}(\varphi(x))$, genera la $G$-topología de $\mathcal{L}(E, F)$.

Ejemplo:

Si E,F son normados entonces la familia $\mathcal{A}$ tiene un solo elemento.

$$
\mathcal{A}=\{1 \mid \|\}
$$




$$
\text { donde }|| c||=\sup _{|| x||_{E} \leqslant 1}|| \rho(x)||_{F}
$$

si se considera $G=\{$ acotados de $E\}$.

Entonces la topología de la convergencia uniforme sobre los acotados es normable.

En el caso particular que $F$ sea el cuerpo. $\mathcal{L}(E, F)=E^{\prime}$, los dos ejemplos siguientes son importantes:

1) La topología de la convergencia simple, que en este caso se anota $\sigma\left(E^{\prime}, E\right)$ y se llama topología débil en $E^{\prime} \cdot\left(E^{\prime}, \sigma\left(E^{\prime}, E\right)\right)$ se llama dual débil de $E$. Se anota $E_{\sigma}^{\prime}$.

2) La topología de la convergencia acotada, que en éste caso se anota $\hat{\beta}\left(E^{\prime}, E\right)$ y se 1 lama topología fuerte en $E^{\prime} \cdot\left(E^{\prime}, B\left(E^{\prime}, E\right)\right)$ se llama dual fuerte de $E$. Se anota $E_{\beta}^{\prime}$.

Observación:

$$
\text { Es claro que } \sigma\left(E^{\prime}, E\right) \leqslant \beta(E, E) \text {. }
$$

§5. ACOTADOS Y EQUICONTINUOS EN $\mathcal{L}(E, F)$.

Consideramos E,F espacios localmente convexos (en esta estación se puede tratar con espacios vectoriales topológicos y los resultados son los mismos, solo debe extenderse la noción de $\mathbf{G}$ - topología a este caso).

Si $\mathcal{L}(E, F)$ tiene una $G$ - topología, una de las necesidades es conocer somo son los conjuntos acotados en esta topología. 
Proposición:

Sea $A \subseteq \mathcal{L}(E, F)$, entonces son equivalentes las siguientes proposiciones:

1) A es acotado en la G - topología

2) Para toda vecindad $V$ de 0 en $F$, el conjunto $\Omega\left\{\varphi^{-1}(V) / \varphi \in A\right\}$ absorve cada $s \in G$.

3) Para cada $S \in G$, el conjunto $U\{\varphi(S) / \varphi \in A\}$ es acotado en $F$. Demostración:

1) $\Rightarrow$ 2) Sin perder generalidad se supone $V$ equilibrado. Como $A$ es acotado entonces $M(S, V)$ absorve $A$ para cada $S \in G$, i.e para cada $s \in G$ existe $\lambda \in \mathbb{I K}$, tal que $\varphi(S) \subseteq \lambda V$ para todo $\varphi \in A$, de aquí que $S \subseteq \lambda \varphi^{-1}(V)$ para todo $\varphi \in A$ entonces $S \subseteq \lambda \quad\left\{\dot{\varphi}^{-1}(V) / \varphi \in A\right\}$.

2) $\Rightarrow 3)$ Sea $s \in G$, si $V$ es una vecindad equilibrada de 0 en $F$, entonces como $S \subseteq \lambda \Omega\left\{\bar{\varphi}^{-1}(V) / \varphi \in A\right\}$. Se tiene que $\mathrm{S} \subseteq \lambda \varphi^{-1}(\mathrm{~V})$ para cada $\varphi \in \mathrm{A}$ luego $\varphi(\mathrm{S}) \subseteq \lambda \mathrm{V}$ para cada $\varphi \in A$ luego $U\{\varphi(S) / \varphi \in A\} \subseteq \lambda V$

$3) \Rightarrow$ 1) $\operatorname{si} S \in G \quad y \quad V$ es una vecindad de 0 en $F$ como $\varphi(S) \subseteq \lambda \mathrm{V}$, algún $\lambda$, para todo $\varphi \in A$ entonces $\varphi \in \lambda \mathrm{M}(\mathrm{S}, \mathrm{V})$, $\varphi \in A$ luego $\mathrm{A} \subseteq \lambda \mathrm{M}(\mathrm{S}, \mathrm{V})$, o sea $\mathrm{A}$ acotado.

Es claro que todo conjunto acotado en la topología de la conver gencia acotada es acotado en la topología simple (simplemente acotado). ¿Cuándo un simplemente acotado es acotado en otra $\mathbf{G}$ - topología?. 
Proposición:

Sean E,F e.l.c. Y G la familia de todos los subconjuntos convexos, equilibrados, acotados y completos de E, entonces todo simplemente acotado es $G$ - acotado. (acotado en la $G$ - topología).

Corolario:

Si E,F son e.l.c. Y E es cuasi - completo entonces los conjuntos acotados son los mismos para cualquier $G$ - topología, donde $G$ es una familia de acotados que cubre $E$.

Un tipo especial de conjuntos resulta siempre ser acotado para todas las $\mathbf{G}$ - topologías, estos son los conjuntos equicontinuos de L $(E, F)$.

Proposición:

Sean $H \subseteq \mathcal{L}(E, F)$ entonces son equivalentes.

1) $\mathrm{H}$ equicontinuo.

2) Para toda vecindad $V$ de 0 en $F, \Omega\left\{\varphi^{-1}(V) / \varphi \in H\right.$ H es una vecindad de 0 en $E$.

3) Para toda vecindad $V$ de $O$ en $F$, existe una vecindad $U$ de 0 en $\mathrm{E}$ tal que $U\{\varphi(V) / \varphi \in H\}$ está contenida en $V$.

Corolario:

Todo equicontinuo de $\mathcal{L}(E, F)$ es acotado para toda $G$ - topuiu gía. 
Demostración:

Sea $V$ una vecindad de 0 en $F$, si se demuestra que $\mathrm{U}=\Omega\left\{\varphi^{-1}(\mathrm{~V}) / \varphi \in \mathrm{H}\right\}$ absorve cada $\mathrm{S} \in \mathrm{G}$, entonces $\mathrm{H}$ es acotado para la G - topología (Ver proposición anterior). Pero como cada $S \in G$ es acotado en $E, S$ es absorvido por toda vecindad de 0 en $E$. Por parte 2 de la proposición, $U$ es una vecindad de 0 en $E$.

Ejemplo:

Sea $E=\left\{f:[0,1] \rightarrow \mathbf{R} / f=0\right.$ en una $v_{0}^{f}$ vecindad de 0$\}$ provisto de la topología uniforme, sea

$$
\mathrm{H}=\left\{\varphi_{\mathrm{n}} \in \mathcal{L}(\mathrm{E}, \mathbb{R}) / \varphi_{\mathrm{n}}(\mathrm{f})=\mathrm{n} \mathrm{f}\left(\mathrm{n}^{-1}\right), \mathrm{n} \in \mathbb{N}\right\}
$$

$H$ es simplemente acotado pero $H$ no es equicontinuo.

Este ejemplo muestra que el recíproco del corolario no vale, ¿cuándo el recíproco se verifica? La siguiente es una respuesta parcial.

Proposición:

Sea E,F e.I.c., E tonelado entonces todo simplemente acotado es equicontinuo.

Demostración:

Sea $H \subseteq \mathscr{L}(E, F)$ simplemente acotado. Sea $V$ una vecindad de $o_{F}$, cerrado, convexa y equilibrada, el conjunto $w=\Omega\left\{\varphi^{-1}(V) / \varphi \in H\right\}$ es cerrado, convexo y equilibrado en $E$. Como $H$ es simplemente acotado, $W$ absorve los conjuntos finitos, en particular $W$ es absorvente luego $W$ es un tonel en $E$ y por lo tanto una vecindad de 0 . 
Otra respuesta parcial.

Proposición:

Sean E,F e.v.t. tal que $E$ es un Baire entonces todo simple mente acotado es equicontinuo.

Corolario:

Sea E,F espacios normados, $E$ tonelado entonces si

$H \subseteq \mathcal{L}(\mathrm{E}, \mathrm{F})$ es puntualmente acotado entonces $\mathrm{H}$ es acotado en la topolo gía de la convergencia acotada (se dice uniformemente acotado usando el lenguaje de Espacios normados).

Este corolario era una proposición originalmente demostrada por Banach - Steinhaus llamado: Principio de la acotación uniforme.

Ligado directamente con este principio se puede estudiar la siguiente

Proposición:

Sean E,F e.l.c., $E$ tonelado si $F$ es un filtro en $\mathcal{L}(E, F)$ simplemente acotado $y$ convergente puntualmente $a \quad f \in F^{E}$ entonces $f \in \mathcal{L}(E, F) \quad y \quad F$ converge a $f$ en la topología de la convergencia pre compacta.

Este es el conocido teorema de Banach - Steinhaus. Aplicaciones de este Teorema pueden encontrarse en F. Tréves, Capítulo 34.

El teorema de la aplicación abierta, (o teorema del gráfico cerrado), el teorema de Hahn - Banach y el teorema de Banach - Steinhaus son los tres resultados fundamentales del Análisis funcional. Existe na turalmente enunciados más generales que los presentados aquí. 
Existen también algunas extensiones de estos teoremas, ver por ejemplo L. Nachbin e Ingleton, el primero para aplicaciones lineales entre dos espacios localmente convexos y el segundo para formas lineales sobre cuerpos valuados no arquimedeanos, esféricamente completos.

Sigue naturalmente, después de estos fundamentos, el estudio de la teoría de la dualidad. Eficaz teoría para los espacios localmente convexos, que tienen un "buen" dual topológico, que consiste, livianamen te hablando, en estudiar propiedades en uno de los espacios para obtener resultados en el espacio dual, vía conjuntos polares. 


\section{BIBLIOGRAFIA.}

[ 1] S. BANACH. Theorie des operators lineaires. Warsav 1932.

[ 2] N. BOURBAKI. Espaces Vectoriels topologiques Ch. 3,4,5. Hermann 1967

[ 3] T. HUSAIN. The open mapping and closed graph Theorems in Topologi cal vector spaces. Oxford Math. Monographs (1965).

[ 4] A. INGLETON. The Hahn - Banuch Theorem for non-Archimedean valued fields. Proc. Camb. Phil. Soc. 48 (1952), 41-45.

[ 5] S. IYAHEN. Barrelled spaces and the open mapping Theorem. J. London. M. Soc. (2) 11 (1975), 421-422.

[ 6] M. MAHOWALD, Barrelled spaces and the closed graph Theorem. J. London. M. Soc. 36 (1961), 108-110.

[ 7] A. MONNA. Functional Analysis in historical perspective. Utrecht. 1973 .

[ 8] L. NACHBIN. A theorem of the Hahn - Banach type for linear transformation, Tran. A.M.S., 68 (1950), 28-46.

[ 9] S. OHWAKI. On Linear operators with closed range. Proc. Japan Acad. 50 (1974), 97-99.

[10] V. PTAK. A Quantitative refinament of the closed graph theorem. Czechoslovak Math J. 24 (99), 1974.

[11] H. SCHAEFER. Topological Vector Spaces. Springer verlag. 1980.

[12] F. TREVES. Topological Vector Spaces, Distributions and Kernels. Academic Press. 1967. 